\title{
Management of Sinonasal Mucormycosis at a Tertiary Care Center: Our Experience
}

\author{
Nikhil M John ${ }^{1}$, Ramabhadraiah Anilkumar $^{2}$, Bandadka Ramya $^{3}$, Hosaagrahara Subbegowda Satish $^{4}$
}

\begin{abstract}
Background: Mucormycosis is a group of invasive infections caused by filamentous fungi of the Mucoraceae family. Most commonly seen in immunocompromised individuals. Sinonasal mucormycosis is a rare infection, difficult to treat, and can also be fatal.

Aims and objectives: To highlight the importance of early detection of disease, early initiation of antifungal therapy, surgical debridement, and adequate management of immunosuppressive conditions.

Materials and methods: In this prospective study, 46 cases of sinonasal mucormycosis with different courses of illness and comorbidities who presented to the ENT OPD at Bengaluru Medical College and Research Institute from July 2015 to June 2018 were included. They were subjected to a common standardized treatment protocol and outcomes were assessed and discussed.

Results: Most of the patients in our study were in the 4th decade of life. Diabetes mellitus was seen in $94 \%$ of our patients. Imaging studies were non-specific with features suggestive of sinusitis. Nasal endoscopy helps in arriving at a provisional diagnosis and knowing the extent of the lesion. Biopsy of tissue showing fungal hyphae with invasion is confirmatory. In our study, 22 (47.8\%) patients expired during the time period. Among the expired patients, only 7 had got combined modality treatment and 20 patients presented after 6 months of the onset of symptoms. In patients with strict glycemic control, no recurrences were seen.

Conclusion: The key to manage mucormycosis is early detection and initiation of antifungal treatment. In any immunocompromised patient presenting with features of sinusitis, mucormycosis has to be ruled out. Liposomal amphotericin B therapy has better compliance. Endoscopic debridement is of vital importance for excellent local control. Control of glycemic status boosts the body's immune process and reduces the chance of recurrence.

Keywords: Endoscopic debridement, Liposomal amphotericin B, Mucormycosis, Sinonasal mucormycosis.

Clinical Rhinology An International Journal (2019): 10.5005/jp-journals-10013-1364
\end{abstract}

\section{INTRODUCTION}

Mucormycosis is a term used to refer to any fungal infection of the order Mucorales, which belongs to the class Zygomycetes. ${ }^{1}$ It was first described by Paulltauf $A$ in 1885 . It can involve the sinonasal tract, upper aerodigestive tract, lungs, gastrointestinal tract, central nervous system, and skin. Sinonasal involvement may progress to form rhinocerebral form in which orbit and brain are also involved. This is the most fatal form of the disease.

It is considered to be one of the most acutely fatal infections known in medical literature if treated inadequately. It is seen commonly in diabetics with uncontrolled glycemic status but can occur in any immunocompromised individual, and on rare occasions can infect normal hosts as well. ${ }^{1}$ The causative agents of mucormycosis are the filamentous fungi of the Mucoraceae family of the order Mucorales, subphylum Mucormycotina. The most frequently isolated species is Rhizopus oryzae followed by Rhizopus microsporus and Absidia corymbifera.

Mucormycosis is most frequently located in the paranasal sinuses (39\%), followed by lungs (24\%), skin (19\%), brain (9\%), gastrointestinal tract (7\%), other organs (6\%), and disseminates in $3 \%$ of cases. Risk factors include poorly controlled diabetes mellitus, diabetic ketoacidosis, other immunocompromised states like organ transplantation, hematologic malignancies, chronic corticosteroid treatment, and hemochromatosis.

Nasal endoscopic examination is the most informative test for possible fungal involvement of nasal cavity and paranasal sinuses. ${ }^{2}$ The mainstay of therapy is the treatment of immunocompromised
${ }^{1-4}$ Sri Venkateshwara ENT Institute, Bangalore Medical College and Research Institute, Bengaluru, Karnataka, India

Corresponding Author: Ramabhadraiah Anilkumar, Sri Venkateshwara ENT Institute, Bangalore Medical College and Research Institute, Bengaluru, Karnataka, India, Phone: +91 9845427714, e-mail: dranil24@yahoo.com

How to cite this article: John NM, Anilkumar R, Ramya B, et al. Management of Sinonasal Mucormycosis at a Tertiary Care Center: Our Experience. Clin Rhinol An Int J 2019;12(2 and 3):52-56.

Source of support: Nil

Conflict of interest: None

state, systemic high dose amphotericin B, and surgical debridement of necrosed or non-viable tissue. ${ }^{3}$

In this study, we attempt to highlight the importance of early detection of sinonasal mucormycosis with the help of clinical features and investigations, the significance of early initiation of antifungal therapy, the necessity for surgical debridement, and most importantly the management of the predisposing immunosuppressed condition for reducing the morbidity and mortality associated with this potentially fatal condition.

\section{Materials and Methods}

This prospective study was conducted at the Department of ENT, Bengaluru Medical College and Research Institute during the time period July 2015 to June 2018. All patients who presented with clinical symptoms and signs suggestive of mucormycosis

(c) The Author(s). 2019 Open Access This article is distributed under the terms of the Creative Commons Attribution 4.0 International License (https://creativecommons.org/licenses/by-nc/4.0/), which permits unrestricted use, distribution, and non-commercial reproduction in any medium, provided you give appropriate credit to the original author(s) and the source, provide a link to the Creative Commons license, and indicate if changes were made. The Creative Commons Public Domain Dedication waiver (http://creativecommons.org/publicdomain/zero/1.0/) applies to the data made available in this article, unless otherwise stated. 
were included in this study. When a patient reported to the hospital detailed history was taken. This included duration of each symptom, relevant history of present and past illness, associated comorbid conditions including diabetes mellitus. Following history, all patients were subjected to thorough local, systemic, and ophthalmologic examination. Routine investigations like blood for hemoglobin percentage ( $\mathrm{Hb} \%)$, total leukocyte count (TC), differential count (DC), erythrocyte sedimentation rate (ESR), urine routine with special emphasis on blood sugar levels [random blood sugar/fasting blood sugar (RBS/FBS), glycated hemoglobin (HbA1c)], urine ketone bodies, arterial blood gas analysis, renal function tests, HIV, and hepatitis B surface antigen ( $\mathrm{HBsAg}$ ) were done in all cases. Diagnostic nasal endoscopic evaluation was done and the extent of the disease was assessed. Radiological investigations such as computed tomography (CT) scan of the nose and para nasal sinuses (PNS) as a preliminary investigation, potassium hydroxide ( $\mathrm{KOH})$ staining, fungal culture, and biopsy of tissue was done in all cases.

All patients were started on intravenous ceftriaxone $1 \mathrm{~g}$ every twelfth hourly and metronidazole $100 \mathrm{~mL}$ every eighth hourly. Adequate hydration was maintained in all cases. Systemic antifungal therapy was started as soon as the diagnosis of mucormycosis was confirmed by biopsy or by culture and after checking blood parameters.

Plain amphotericin B (1-1.5 mg/kg body wt/day) was given in dosage, as per nephrologist opinion. For those with renal impairment and in affordable patients, liposomal amphotericin B (5-7.5 mg/kg body wt/day) was started in renal modified dosages. In addition, topical antifungal therapy with $50 \mathrm{mg}$ vial of intravenous amphotericin $B$ and $10 \mathrm{~mL}$ of sterile water as nasal drops, in a dosage of $4 \mathrm{~mL}$ to each nostril 4 times daily, was given in all cases.

Surgical debridement of the lesion under general anesthesia was done in the majority of the cases and repeated as required. Antifungal therapy was continued postoperatively for 6 weeks in most of the patients. All patients were kept under stringent follow-up with weekly rigid nasal endoscopy till the first 4 weeks. Regular saline douching was also advised in all cases. Apart from this, correction of hyperglycemic status as per physician advice was strictly followed.

\section{Results}

The total number of patients included during this time period was 46. Male patients $(n=26)$ were more compared to female patients $(n=20)$. The male to female ratio was 1.3:1. The majority of the patients were in the 4 th decade $(n=25)$. This was followed by patients from the 5th decade $(n=13)$ and 6th decade of life $(n=$ 5) (Fig. 1). These patients presented with various symptoms. Nasal discharge and nasal obstruction were the most common presenting complaints. Fever was present in only 17 (36.9\%) patients (Table 1). Oral cavity examination showed erosion of hard palate in 9 (19.5\%) patients (Fig. 2). The various comorbidities among our patients were noted from history, clinical examination, and laboratory investigations. Diabetes mellitus was found to be present among the majority of our patients followed by ketoacidosis and renal impairment (Fig. 3). The radiological investigation included a CT scan of the nose and PNS. However, the findings were inconclusive. The majority of the cases $(91.3 \%)$ showed mucosal thickening involving the sinuses. Soft tissue opacity resembling polyposis was the next common finding. In advanced cases, bone erosion was seen (Fig. 4).
All patients underwent nasal endoscopy which helped assess the extent of the lesion. Examination showed blackish crusts filling the nasal cavity and middle meatal region in 43 patients (93.4\%). Histopathological examination of the tissue was done in all cases which showed broad, branched ribbon-like aseptate hyphae with evidence of angioinvasion and tissue necrosis which confirmed the diagnosis (Fig. 5). Antifungal therapy was commenced after the biopsy report.

Among the 46 patients, 30 patients were treated with a combined modality consisting of antifungal therapy and endoscopic surgical debridement. Surgical debridement was done till signs of viability were seen (Fig. 6). Orbital exenteration was done in three of our patients. In the case of hard palatal perforation which was noticed in nine cases, the palate was removed and reconstruction with palatal obturator placement was done. In 16 patients, only antifungal therapy was started as these patients were not stable and fit for surgery.

During the study period, 22 patients (47.8\%) expired among which only 7 had got combined modality treatment. Among the patients who expired 20 (90.9\%) presented after 6 months of the onset of symptoms.

Of the 24 patients who survived, 12 were on liposomal amphotericin B ( 5 patients only antifungal and 7 were on combined modality). After discharge, they had no further recurrence till 3 months of follow-up. Twelve patients were on plain amphotericin $B$ (six patients only antifungal and six were on combined modality). Five patients discontinued treatment. On the first month of follow-up, five patients showed recurrence. They were readmitted

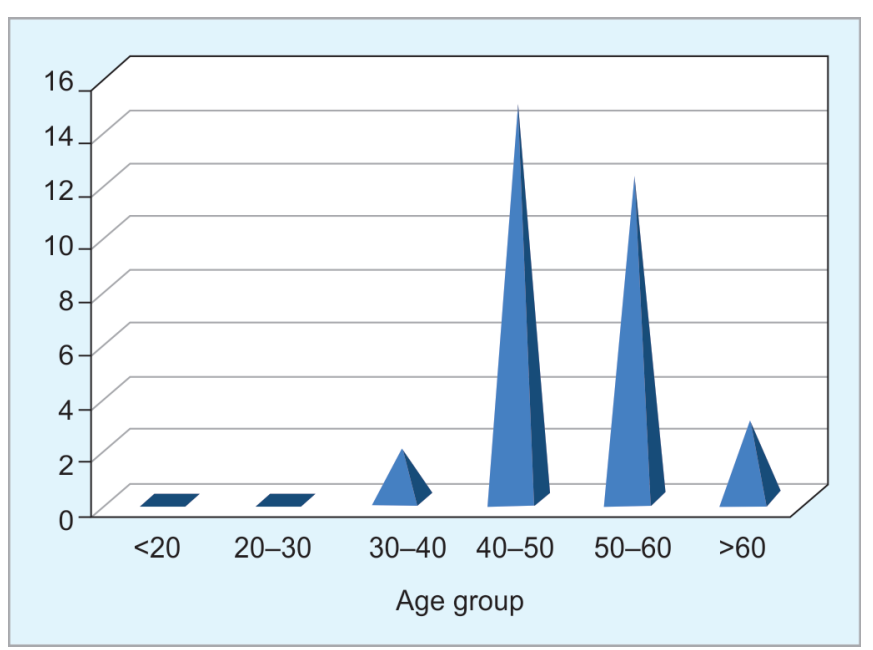

Fig. 1: Age distribution among the patients

Table 1: Frequency of symptoms

\begin{tabular}{ll}
\hline Symptoms & Frequency \\
\hline Fever & 14 \\
Nasal discharge & 28 \\
Nasal obstruction & 27 \\
Facial/orbital swelling & 29 \\
Visual disturbance & 26 \\
Odynophagia & 16 \\
Others (mental disturbance, etc.) & 4 \\
\hline
\end{tabular}



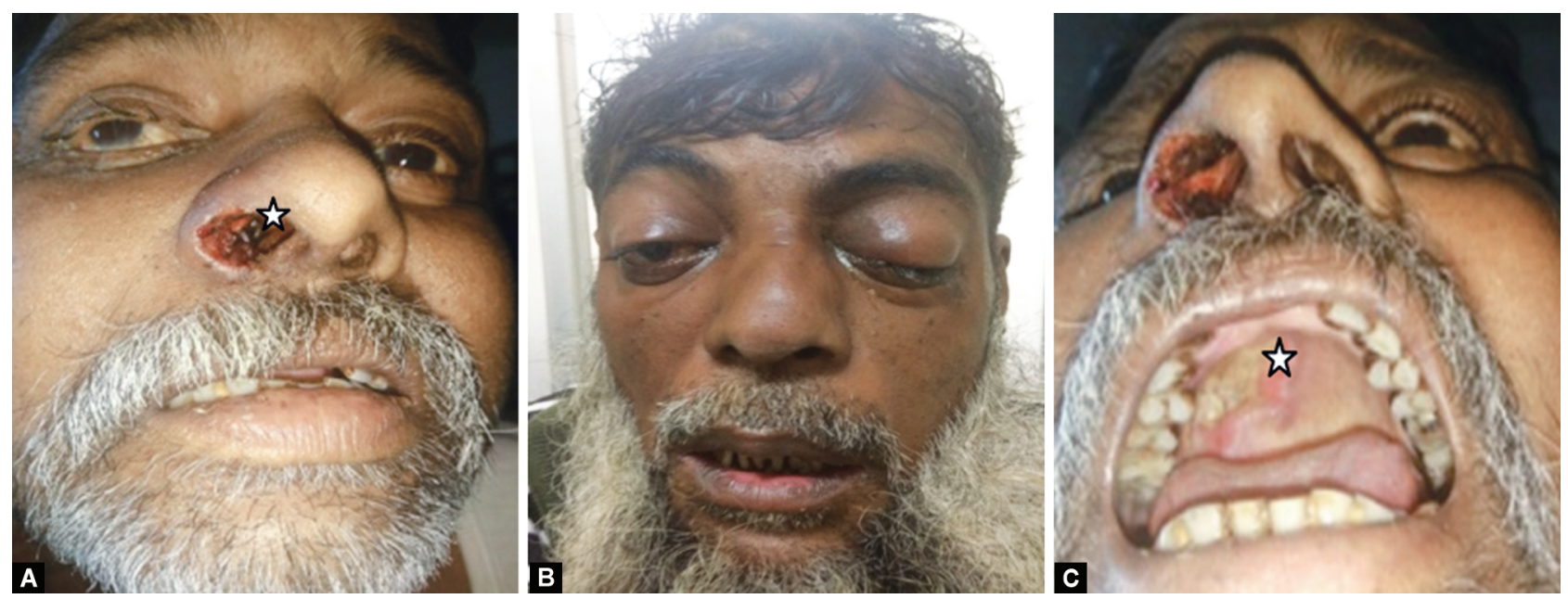

Figs 2A to C: Clinical features: (A) Nasal crust; (B) Periorbital swelling; (C) Hard palate erosion

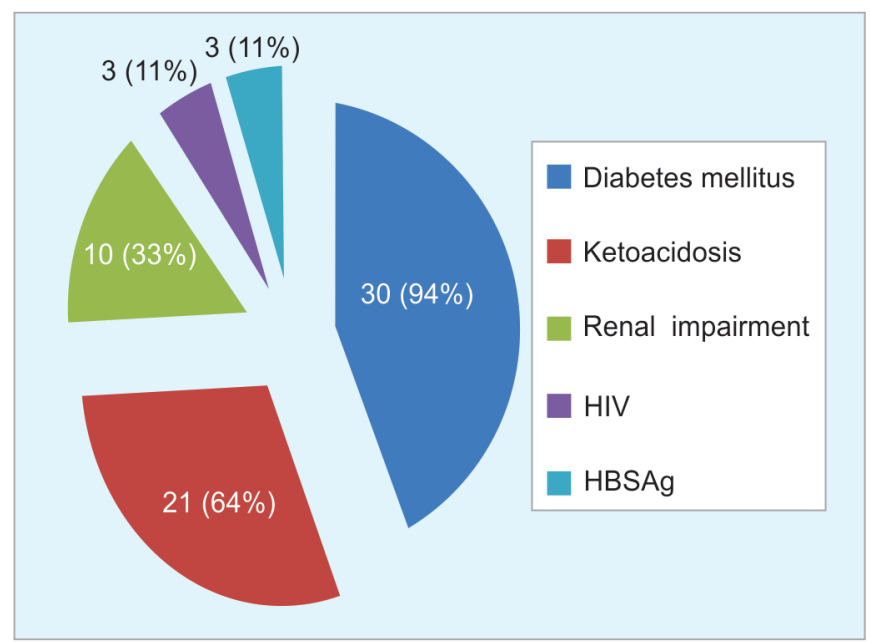

Fig. 3: Risk factors among the patients

and started on liposomal amphotericin B with strict glycemic control.

\section{Discussion}

Mucormycosis is one of the most rapidly progressive lethal infections having a mortality of $70-100 \% .{ }^{4}$ In our study, male patients were more compared with female patients ( $M: F=1.3: 1)$. Most of the patients in our study were in the 4 th decade $(54.3 \%)$. This was followed by patients from the 5 th decade $(28.2 \%)$ and 6 th decade of life (10.8\%). This was similar to the study conducted by Roden et al., ${ }^{5}$ in which the median age of patients was 40 years.

Our patients presented with multiple symptoms which included fever, sinus tenderness, facial swelling, periorbital swelling, nasal discharge, nasal obstruction, and visual disturbance. A study on mucormycosis by Parfrey ${ }^{6}$ and Ferry and Abedi $^{7}$ showed similar complaints in patients with mucormycosis. The initial symptom was similar to sinusitis in the majority of our patients (97\%). Fever was present in only 17 (36.9\%) cases in our study, similar to the study conducted by Talmi et al. ${ }^{8}$ in which fever was present in less than half of the cases. Several studies have shown that about $70 \%$ of sinonasal cases were found in diabetic patients with ketoacidosis. ${ }^{9}$ In a study on 126 patients with rhinocerebral mucormycosis by
Strasser et al., ${ }^{10}$ he observed that $70 \%$ of patients were diabetic. Our study had consistent findings with 30 of our patients being diabetics and 21 being in ketoacidosis. The high association of mucormycosis in diabetes mellitus and ketoacidosis might be due to the innate active ketone reductase system present in Rhizopus organisms which helps it to thrive in high glucose, acidotic conditions. Also, there is increased availability of serum iron, decreased phagocytic and chemotactic activity of neutrophils in these conditions.

The initial imaging study in mucormycosis is a CT scan of the nose and PNS. However, the findings are usually non-specific with features suggestive of sinusitis or polypoidal soft tissue changes. In our study, sinus mucosal thickening (91.3\%) and polypoidal changes (54.3\%) were the most common findings. A study conducted by Singh et al. ${ }^{11}$ showed unilateral sinus mucosal thickening as the most consistent finding. In advanced stages, we also observed osteolytic changes involving sinus walls and heterogeneous opacification with an air-fluid level. This shows that in the initial stages it is not wise to rely entirely on imaging studies to rule out mucormycosis. magnetic resonance imaging (MRI) is the appropriate radiological investigation when there is a suspicious cavernous sinus/intracranial involvement. $^{2}$

Nasal endoscopy has an important role to arrive at a provisional diagnosis and also to know the extent of the lesion. Endoscopic examination showing discoloration, ulceration involving nasal mucosa, and turbinates with eschar formation is highly suggestive of mucormycosis. In our study, we observed blackish crusts filling the nasal cavity and middle meatal region in the majority 43 (93.4\%) of the cases. Also, erosion of the hard palate was seen in nine (19.5\%) cases. Gillespie and O'Malley ${ }^{12}$ in their study observed that $80 \%$ of patients developed a necrotic lesion on either the nasal or oral mucosa.

Twenty-two of our patients expired during the time period among which 20 patients presented after 6 months of the onset of symptoms. A study conducted by Chamilos et al. ${ }^{13}$ and Spellberg and Ibrahim ${ }^{14}$ showed that initiation of antifungal therapy within 5 days of diagnosis/onset of symptoms had better prognosis when compared with late initiation of treatment, i.e., 83 vs $49 \%$ survival. In patients with a high index of suspicion of mucormycosis antifungal therapy can be started, following a positive $\mathrm{KOH}$ mount, after obtaining written informed consent before the biopsy or fungal culture reports confirm the diagnosis. 

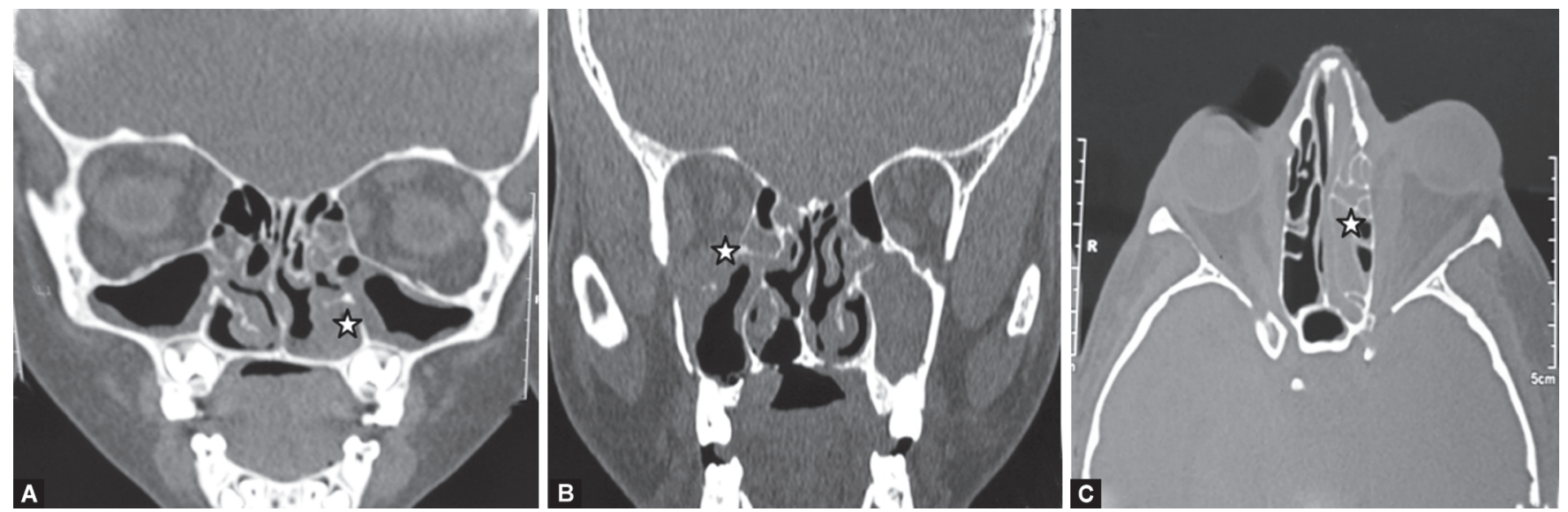

Figs 4A to C: CT PNS showing sinus opacification with bone erosion
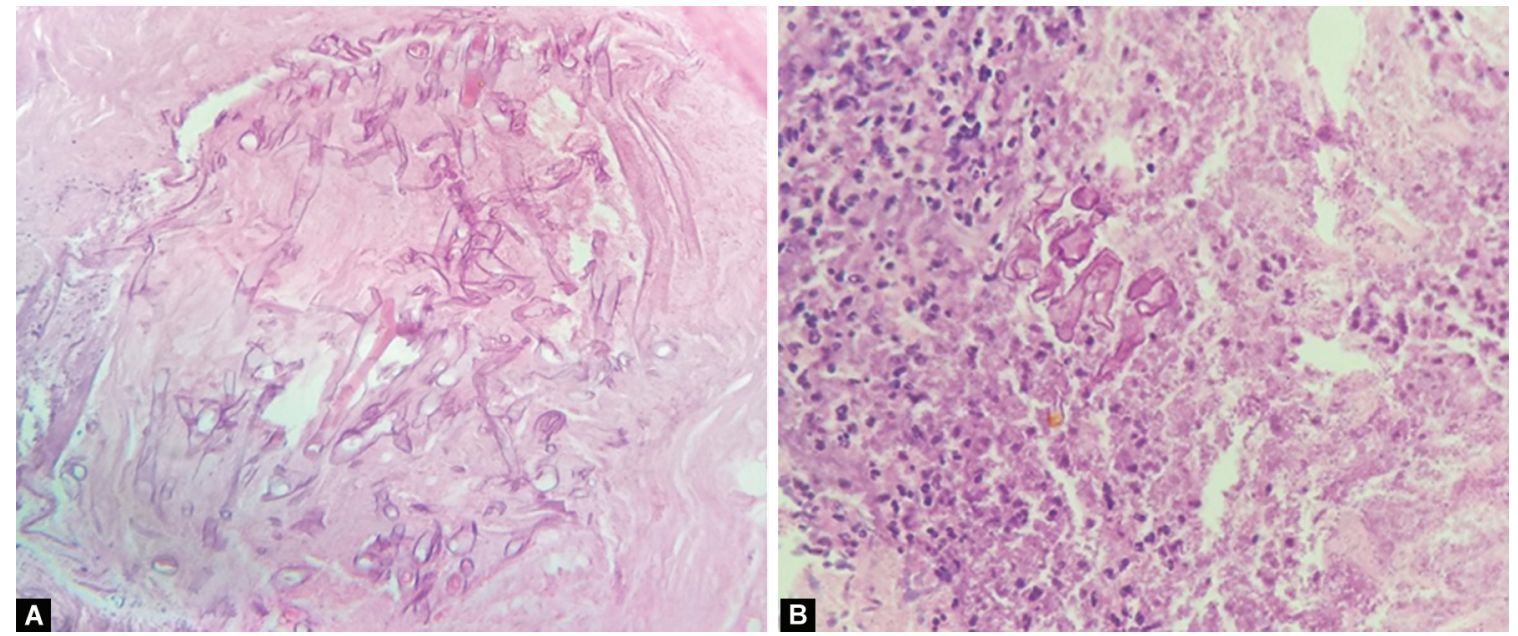

Figs 5A and B: Histopathological examination showing broad ribbon like aseptate hyphae confirming mucormycosis

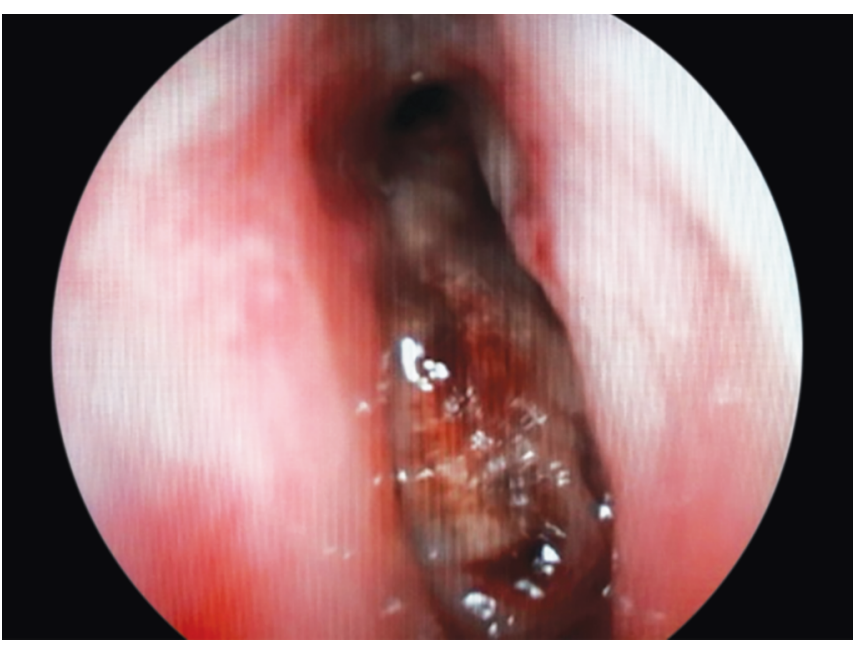

Fig. 6: Endoscopic image during surgical debridement of crusts

One patient with recently detected type 2 diabetes mellitus and features of pansinusitis referred to us due to no improvement in symptoms despite higher antimicrobial therapy showed a normal nasal endoscopic examination. A hard palate ulcer was noted but a biopsy is done elsewhere reported as squamous metaplasia.
Due to a high index of suspicion, a deep debridement was done following which the histopathological examination was positive for mucormycosis. The patient was immediately started on liposomal amphotericin B. After 4 weeks of treatment, a repeat biopsy was negative and the patient was discharged symptom-free. This shows the importance of a high index of suspicion for mucormycosis in immunocompromised patients with features of sinusitis when there is no relief with usual medications.

In our study, liposomal amphotericin B was started in 14 patients, among which 12 survived. Liposomal amphotericin B was found to be less nephrotoxic with no drug reactions than plain amphotericin B. A study on mucormycosis by Spellberg and Ibrahim ${ }^{14}$ and Gleissner et al. ${ }^{15}$ showed that treatment with liposomal amphotericin B was associated with a $67 \%$ survival rate, compared with $39 \%$ survival in patients treated with plain amphotericin B. In this study, a faster healing rate was observed in patients started on liposomal amphotericin B when compared with plain amphotericin B. Moreover, eight patients who survived showed no recurrence after 6 months of follow-up.

Endoscopic sinonasal debridement under general anesthesia was done in 30 patients along with systemic antifungal therapy. Debridement of the involved structures was extended until clear bleeding margins were seen, this helps in better availability of antifungals to the site and results in thorough disease clearance. 
Orbital involvement requires exenteration which was performed in three of our patients.

The three patients who showed recurrence on follow-up had poor glycemic control. This stresses the importance of strict glycemic control in the management of mucormycosis.

\section{Conclusion}

Sinonasal mucormycosis is an extremely fatal disease that has to be promptly managed as an ENT emergency. In any immunocompromised patient presenting with features suggestive of sinusitis, mucormycosis has to be ruled out. The key factor in its management is early detection and initiation of antifungal treatment. Liposomal amphotericin B therapy though more expensive is found to be safer, more effective with less toxic reactions, recurrence rate, and faster recovery than plain amphotericin B. Endoscopic debridement is of vital importance as it leads to excellent local control, reduces morbidity and mortality associated with the disease. In addition to a specific treatment, aggressive control of the immunocompromised state is vital as it helps in recovery by boosting the body's immune process and also reduces the chance of recurrence.

\section{References}

1. Ferguson BJ. Mucormycosis of the nose and paranasal sinuses. Otolaryngol Clin North Am 2000;3(2):349-365. DOI: 10.1016/S00306665(00)80010-9.

2. Epstein VA, Kern RC. Invasive fungal sinusitis and complications of rhinosinusitis. Otolaryngol Clin North Am 2008;41(3):497-524. DOI: 10.1016/j.otc.2008.01.001.

3. Auluck A. Maxillary necrosis by mucormycosis. A case report and literature review. Med Oral Patol Oral Cir Bucal 2007;12(5):E360-E364.

4. Jagdish C, Reetika S, Pallavi S, et al. Mucormycosis of the paranasal sinus with gas forming maxillary osteomyelitis - a case report. Internet J Microbiol 2012;10(1). Available from: https://ispub.vom/ IJMB/10/1/13760.
5. Roden MM, Zaoutis TE, Buchanan WL, et al. Epidemiology and outcome of Zygomycosis: a review of 929 reported cases. Clin Infect Dis 2005;41(5):634-653. DOI: 10.1086/432579.

6. Parfrey NA. Improved diagnosis and prognosis of mucormycosis. A clinicopathologic study of 33 cases. Medicine 1986;65(2):113-123. DOI: 10.1097/00005792-198603000-00004.

7. Ferry AP, Abedi S. Diagnosis and management of rhinoorbito-cerebral mucormycosis (phycomycosis). A report of 16 personally observed cases. Ophthalmology 1983;90(9):1096-1104. DOI: 10.1016/S01616420(83)80052-9.

8. Talmi YP, Goldschmied-Reouven A, Bakon M, et al. Rhino - orbital and rhino-orbito-cerebral mucormycosis. Otolaryngol Head Neck Surg 2002;127(1):22-31. DOI: 10.1067/mhn.2002.126587.

9. McNulty JS. Rhinocerebralmucormycosis: predisposing factors. Laryngoscope 1982;92:1140-1143.

10. Strasser MD, Kennedy RJ, Adam RD. Rhinocerebralmucormycosis: therapy with amphotericin B lipid complex. Arch Intern Med 1996;156(3):337-339. DOI: 10.1001/archinte.1996.00440030 145018.

11. Singh I, Gupta V, Gupta SK, et al. Our experience in endoscopic management of mucormycosis: a case series and review of literature. Int J Otorhinolaryngol Head Neck Surg 2017;3(2):465-471. DOI: 10.18203/issn.2454-5929.ijohns20171217.

12. Gillespie MB, O'Malley BW. An algorithmic approach to the diagnosis and management of invasive fungal rhinosinusitis in the immunocompromised patient. Otolaryngol Clin North Am 2000;33(2):323-334. DOI: 10.1016/S0030-6665(00)80008-0.

13. Chamilos G, Lewis RE, Kontoyiannis DP. Delaying amphotericin Bbased frontline therapy significantly increases mortality among patients with hematologic malignancy who have zygomycosis. Clin Infect Dis 2008;47(4):503-509. DOI: 10.1086/590004.

14. Spellberg B, Ibrahim AS. Recent advances in the treatment of mucormycosis. Curr Infect Dis Rep 2010;12(6):423-429. DOI: 10.1007/ s11908-010-0129-9.

15. Gleissner B, Schilling A, Anagnostopolous I, et al. Improved outcome of zygomycosis in patients with hematological diseases? LeukLymphoma 2004;45(7):1351-1360. DOI: 10.1080/10428190310001653691. 